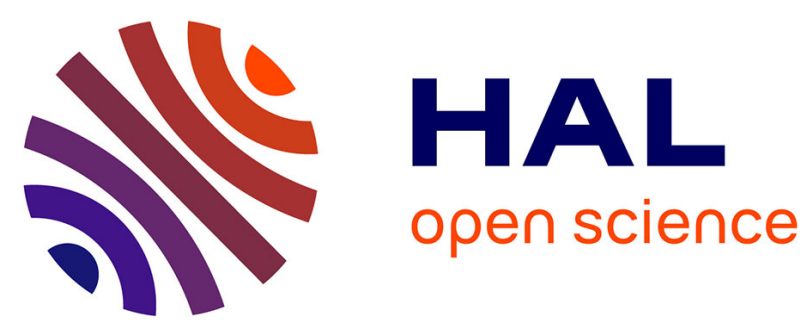

\title{
Ultrafast dynamics of photoexcited Hot carrier generation and injection in AgNWs@TiO2@GNS nanostructures
}

Yiwen Li, Jiaji Chen, Junzi Li, Marie-Hélène Delville, Mona

Tréguer-Delapierre, Tingchao He, Rui Chen, Zikang Tang

\section{To cite this version:}

Yiwen Li, Jiaji Chen, Junzi Li, Marie-Hélène Delville, Mona Tréguer-Delapierre, et al.. Ultrafast dynamics of photoexcited Hot carrier generation and injection in AgNWs@TiO2@GNS nanostructures. Journal of Physical Chemistry C, 2018, 122 (26), pp.14857-14864. 10.1021/acs.jpcc.8b05017 . hal01830872

\section{HAL Id: hal-01830872 \\ https://hal.science/hal-01830872}

Submitted on 29 Jan 2021

HAL is a multi-disciplinary open access archive for the deposit and dissemination of scientific research documents, whether they are published or not. The documents may come from teaching and research institutions in France or abroad, or from public or private research centers.
L'archive ouverte pluridisciplinaire HAL, est destinée au dépôt et à la diffusion de documents scientifiques de niveau recherche, publiés ou non, émanant des établissements d'enseignement et de recherche français ou étrangers, des laboratoires publics ou privés. 


\title{
Ultrafast Dynamics of Photoexcited Hot Carrier Generation and Injection in AgNWs@ $\mathrm{TiO}_{2} @$ GNS Nanostructures
}

Yiwen $\mathrm{Li}^{1,2}$, Jiaji Cheng ${ }^{3}$, Junzi $\mathrm{Li}^{3}$, Marie-Hélène Delville ${ }^{4}$, Mona Tréguer-Delapierre ${ }^{4}$, Tingchao $\mathrm{He}^{3, *}$, Rui Chen ${ }^{2, *}$, and Zikang Tang ${ }^{1, *}$

${ }^{1}$ The Institute of Applied Physics and Materials Engineering, University of Macau, Avenida da Universidade, Taipa, Macau, P. R. China

2 Department of Electrical and Electronic Engineering, Southern University of Science and Technology, Shenzhen, Guangdong 518055, P. R. China

${ }^{3}$ College of Physics and Energy, Shenzhen University, Shenzhen, Guangdong 518060, P. R. China

${ }^{4}$ Institut de Chimie de la Matière Condensée de Bordeaux - CNRS, 87, avenue du Dr Schweitzer 33608 Pessac Cedex, France

\begin{abstract}
The generation and injection of hot electrons in plasmonic nanostructures have received a wide-range potential application in the next generation of ultrafast nanophotonics as well as energy harvesting, storage and conversion. In many cases, the energetic carriers with high energy beyond Fermi level are desirable for photochemical reactions due to the tremendous efficient hot electron injection. However, energetic electrons have up to now hardly been observed and they generally suffer from a low generation efficiency in conventional plasmonic nanostructures. This paper reports on the fabrication of hybrid $\mathrm{AgNWs} @ \mathrm{TiO}_{2} @ \mathrm{Au}$ core-shell nanostructures and the investigation of the ultrafast dynamics of their energetic carriers based on interband and intraband excitations through transient absorption spectroscopy measurements. Finally, the different electronic transition
\end{abstract}


processes are discussed in terms of ultrafast decay response, which is necessary to design proper nanostructures aimed at efficient hot electron injection.

Keywords: Ultrafast Carrier Dynamics; Interband Excitation; Intraband Excitation; Hot Electrons; Transient Absorption. 


\section{INTRODUCTION}

Surface plasmon, which derives from the collective oscillation of free conducted electrons in metallic nanoparticles (NPs), contributes to many tremendous advances in nanoscience. ${ }^{1}$ These nanoparticles were used in many different areas including optoelectronics and sensors, which are inspired by their superior optical behavior. ${ }^{2-5}$ After incident light excitation with resonant energy, electrons undergo a decay through either a radiative channel via scattering, or a non-radiative pathway via electron-hole interaction. ${ }^{6-7}$ In particular, the generation and injection of hot electrons in electron-hole pairs are significant for photochemical applications which involve catalysis and photodetectors. $^{8-11}$ Generally speaking, nonthermal electrons induced from plasmonic decay could establish a non-Fermi distributed excited carrier population with indefinable temperature through electron-electron scattering processes accompanied with electron thermalization. ${ }^{12}$ The timescale is around several hundred femtoseconds. The next steps then imply a further cooling process to break the hot thermalized distribution. The corresponding relaxation is induced by an electron-phonon coupling with a timescale of a few picoseconds. ${ }^{13}$ Ultrafast dynamics study through ultrafast transient spectroscopy is then essential to examine plasmonic induced hot electron injection or energy transfer between plasmon and semiconductor. ${ }^{14-16}$

In the past decades, ordinary nanostructures such as plasmonic metal@metal oxide core-shell nanostructures have been studied through time-resolved optical measurements to discuss the charge or energy transfer dynamics. ${ }^{16-18}$ Besides, researchers have observed the anomalous pulsewidth-limited relaxation induced by efficient scattering among energetic hot electrons in complicated plasmonic nanostructures. ${ }^{19-20}$ Efficient hot electron generation and energy flow are strongly determined by the excitation energy and configuration of the nanostructures such as the nature of the metal and the dielectric layer thickness. However, most of these nanostructures were 
fabricated with top-down methods such as electron-beam lithography, during which high cost laborious manipulation is unavoidable. Colloidal wet chemistry can provide a facile approach to a wide variety of NPs synthesis in terms of compositions, sizes, shapes, core-shell structures and complexity. ${ }^{21-22}$ Versatile and scalable hybrid plasmonic nanostructures can then be obtained without relying on high cost instruments like other nanofabrication methods. Moreover, the synthetic process has no limitation for arbitrary materials besides metallic nanoparticles. ${ }^{23}$

Here, desirable plasmonic semiconductor hybrid nanostructures based on silver nanowires (AgNWs) coated with a well-controlled thickness $\mathrm{TiO}_{2}$ shell were prepared using an overall wet chemistry approach. For subsequent investigation, gold nanoparticles (GNPs) were further grown onto $\mathrm{TiO}_{2}$ to form a gold nanoshell (GNS) with different thicknesses. Hot carrier dynamics from photoexcited and plasmonic electrons induced through interband and intraband transitions were investigated, and a pulsewidth-limited relaxation induced by electron-electron scattering was observed. Our study shows how the interband and intraband excitations could affect the electron transition process in plasmonic nanostructures. These results provide physical insights into hot electron conditions in multicomponent systems. The observed photo-physical process is closely related to hot electron injection, and it is therefore essential in order to understand more in details the excited state carrier dynamics of nanostructures and to develop the most adapted ones for hot electron devices. $^{24-25}$

\section{EXPERIMENTAL SECTION}

Materials: Anhydrous ethylene glycol (99.8\%), platinum chloride $\left(\mathrm{PtCl}_{2}, 99.99+\%\right)$, Silver nitrate $\left(\mathrm{AgNO}_{3}, 99 \%\right)$, Poly(vinyl pyrrolidone) (PVP, MW 55 000), Absolute ethanol (99.9\%, Scharlau), Titanium(IV) tetraisopropoxide (TTIP, 97\%, Aldrich), Acetyl acetone (Acac, 99\%, Aldrich), 
(3-Aminopropyl) triethoxysilane (APTES, 99\%, Aldrich), Gold(III) tetra chloric acid trihydrate $\left(\mathrm{HAuCl}_{4} \cdot 3 \mathrm{H}_{2} \mathrm{O}, 99 \%\right.$, Aldrich) and 11-Mercaptoundecanoic acid (MUA, 98\%, Aldrich) were used without further purification. Ultrapure water $\left(18.2 \mathrm{MOhm} \mathrm{cm}\right.$ at $\left.25^{\circ} \mathrm{C}\right)$ was obtained with a Direct-Q3 system (Millipore).

Controlled Deposition of $\mathrm{TiO}_{2}$ on AgNWs: The deposition of $\mathrm{TiO}_{2}$ on the $\mathrm{AgNW}$ s surface was performed using acetyl acetone as a retardant reagent to control the hydrolysis and polycondensation of the titanium precursor. In an optimised synthesis, a total $23 \mathrm{~mL}$ solution including: $20 \mathrm{~mL}$ of the as-synthesized MUA functionalized AgNWs $(0.08 \mathrm{mg} / \mathrm{mL})$ in pure ethanol and a mixture of $0.25 \mathrm{~mL}$ of Milli-Q water and $2.75 \mathrm{~mL}$ absolute ethanol were introduced in a $50 \mathrm{~mL}$ flat-bottom flask with stirring as a template solution. In parallel, $1 \mathrm{~mL}$ of the precursor solution at a concentration of 14 mM TTIP mixed with Acac (1:2 molar ratio) was prepared. The precursor solution was then diluted with various fractions of pure ethanol. The thickness of the $\mathrm{TiO}_{2}$ coating was effectively dependent on the degree of dilution, typically to obtain 5, 10, 20, 50 and $70 \mathrm{~nm}$ thickness of $\mathrm{TiO}_{2}$, the precursor solution must be diluted $10,5,4,3$ and 2 times respectively via addition of pure ethanol. $1 \mathrm{~mL}$ of either of these light yellow solutions was then injected into the template solution at a rate of $1 \mathrm{~mL} / \mathrm{h}$ with a syringe pump. After the completion of the injection, the resulting colloidal suspension was heated at to $80{ }^{\circ} \mathrm{C}$ under stirring for $1.5 \mathrm{~h}$ for the condensation procedure to be completed. The colloidal suspension was then washed by 5 extensive centrifugation cycles with pure EtOH and eventually stored in $25 \mathrm{~mL}$ pure ethanol at a concentration of approximately $0.5,0.8,1.4,1.9$ and 2.1 $\mathrm{mg} / \mathrm{mL}$ for ca. $5,10,20,50$ and $70 \mathrm{~nm} \mathrm{TiO}_{2}$ thickness samples respectively. The obtained colloidal suspensions hereafter named as $\mathrm{AgNWs} @ \mathrm{TiO}_{2}$ are then ready for the next step. In any case, for large production, the reaction can simply be scaled up to 10-20 times. 
Synthesis of AgNWs@TiO,@APTES-GNPs: Typically, $0.1 \mathrm{~mL}, 10.88 \times 10^{3} \mathrm{nM}$ of as-synthesized APTES-GNPs was diluted ten times with $0.9 \mathrm{~mL}$ of pure water and sonicated for $10 \mathrm{~min}$. before use. Then $1 \mathrm{~mL}, 0.1 \mathrm{mg} / \mathrm{mL}$ AgNWs $@ \mathrm{TiO}_{2}$ was added to the APTES-GNPs solution with $15 \mathrm{~min}$. sonication. The mixture was kept overnight at room temperature in a roller-mixer at a rate of 250 $\mathrm{rpm} / \mathrm{min}$. Then, the mixture was washed 3 times with pure water, precipitated by centrifugation and eventually kept in $2 \mathrm{~mL}$ pure water at a concentration of $\sim 0.06 \mathrm{mg} / \mathrm{mL}$. The obtained colloidal suspension hereafter named as AgNWs@TiO ${ }_{2} @$ APTES-GNPs is then ready for the next step. For large production, the reaction can be simply scaled up to 10-20 times.

Step by Step Regrowth of APTES-GNPs for Uniform GNS Formation: After obtaining AgNWs@TiO ${ }_{2} @$ APTES-GNPs, a layer of GNS could then be achieved by a seed-mediated step by step growth of APTES-GNPs. To do this, a gold precursor solution was prepared, in which $8 \mathrm{~mL}, 25$ $\mathrm{mM}$ of $\mathrm{HAuCl}_{4}$ was added to $92 \mathrm{~mL}$ of pure water with $300 \mathrm{mg}$ of $\mathrm{K}_{2} \mathrm{CO}_{3}$. The solution was stirred for $30 \mathrm{~min}$. to obtain a colourless solution and kept at $4^{\circ} \mathrm{C}$ for two days before any use. Then in a typical run of regrowth of GNS, $1 \mathrm{~mL}$ of this gold precursor was added to $23 \mathrm{~mL}$ solution consisted of $4 \mathrm{~mL}, 0.06$ mg/mL AgNWs@TiO ${ }_{2} @$ APTES-GNPs, 19 mL pure water and $10 \mathrm{~mL}, 10 \mathrm{~g} / \mathrm{L}$ PVP ( 29,000 MW). After $1 \mathrm{~min}$. vortex in a falcon, $50 \mu \mathrm{L}$ of formaldehyde was added to the solution and then kept in roller-mixer overnight at a rate of $250 \mathrm{rpm} / \mathrm{min}$. Afterwards, the solution was washed 4 times with pure water precipitated by centrifugation and eventually kept in $4 \mathrm{~mL}$ pure water for TEM observation and UV-vis. characterizations. To further grow the GNS, such an experiment can be run repeatedly until the desired layer is achieved and typically, with 4 times regrowth a thin layer of gold can be obtained.

One-Pot Regrowth of APTES-GNPs for Uniform GNS Formation: As indicated from previous experimental details, for $4 \mathrm{~mL}$ of 0.06 mg/mL AgNWs@ $\mathrm{TiO}_{2} @$ APTES-GNPs, $4 \mathrm{~mL}$ of gold 
precursor is needed for a thin layer formation. In this way, for a one-pot regrowth of APTES-GNPs, a $4 \mathrm{~mL}$ gold precursor solution $(2 \mathrm{mM})$ was directly added to $4 \mathrm{~mL} 0.06 \mathrm{mg} / \mathrm{mL}$ AgNWs@TiO ${ }_{2} @$ APTES-GNPs with 16 mL pure water and 10 mL, 10 g/L PVP ( 29,000 MW) while other experimental conditions were kept unchanged. Then, the shell thickness could be controlled by tuning the amount of gold precursor. With $4,8,12$, mL of gold precursor $(2 \mathrm{mM})$, the corresponding thickness of GNS were around 30, 45, $60 \mathrm{~nm}$ respectively.

Characterization. Transmission electron microscope (TEM) characterizations were performed with a JEOL JEM-1400 Plus microscope operating at $120 \mathrm{kV}$. The samples were prepared as follows: colloids were diluted in ethanol and one drop of the diluted suspension was deposited on a copper grid coated with a carbon membrane. Chemical analysis was carried out by STEM coupled to EDX were acquired with a JEOL 2200 FS equipped with a field emissive gun, operating at $200 \mathrm{kV}$ and with a point resolution of $0.23 \mathrm{~nm}$. The UV-vis measurement is performed with a TU-1901 double-beam UV/Vis spectrophotometer (Beijing Purkine General Instrument Co. Ltd., China)

Optical Simulation: The simulated extinction spectra and field enhancement were performed using a three-dimensional module on Finite-difference time-domain (FDTD) based software (Lumerical Solutions). The model systems include a three-dimensional nanoparticle surrounding with water and the dielectric function data for silver and gold taken from Johnson and Christy.

Transient Absorption Measurements: In this measurement spectra-Physics Solstice were utilized as the ultrafast laser beams with a regeneratively amplified Ti: sapphire laser system (Coherent Legend, $800 \mathrm{~nm}, 100 \mathrm{fs}$ and $1 \mathrm{kHz}$ repetition rate). Part of the $800 \mathrm{~nm}$ output laser was guided into Traveling Wave Optical Parametric Amplifier (TOPAS) to generate pulse for pump with desirable wavelength. The power of the pump pulses could be controlled with a series of neutral-density filter wheels. The pump beam was focused at the sample with a spot diameter about $200 \mu \mathrm{m}$ as following 
chopped by as synchronized chopper at $333 \mathrm{~Hz}$. The probe beams in the wavelengths between 350 and $800 \mathrm{~nm}$ were achieved by supercontinuum generation from a thin $\mathrm{CaF}_{2}$ plate. The probe pulses were focused into a fiber-coupled multichannel spectrometer with complementary metal-oxide-semiconductor (CMOS) sensors with detecting at a frequency of $1 \mathrm{kHz}$. The delay between the pump and probe pulses was controlled by a motorized delay stage. The aqueous samples were dispersed without bubble in $1 \mathrm{~mm}$ cuvette and both beams of pump and probe lasers were spatially overlapped onto cuvette.

\section{RESULTS AND DICUSSION}

\section{Morphology and Steady State Optical Measurements of Hybrid Nanostructures}

The $35 \mathrm{~nm}$ diameter with $3 \mu \mathrm{m}$ length AgNWs were employed for the synthesis of AgNWs@TiO ${ }_{2} @$ GNPs which was performed as illustrated in Figure 1 for a $\mathrm{TiO}_{2}$ shell of $5 \mathrm{~nm}$. After that, GNPs were continuously grown to form GNS leading to AgNWs@ $\mathrm{TiO}_{2} @ \mathrm{GNS}$ core-shell-shell hybrid nanostructures. To achieve this, gold precursor in water was added to AgNWs@TiO ${ }_{2} @$ GNPs and then reduced slowly to grow the GNPs in each run as shown in the experimental method. The TEM images shown in Figs. S1c-f confirm the step-by-step growth of the GNPs and the formation of GNS after four cycles. The corresponding UV-vis spectra (Figure S1g) witness that the characteristic plasmon peak of GNPs undergoes an obvious red shift from 538 to $592 \mathrm{~nm}$. The thickness of the GNS is measured to be around $30 \mathrm{~nm}$ based on more than 100 acquisitions. The distribution of silver, titania and gold within the nanostructures was further investigated by energy-dispersive X-ray spectroscopy (EDX) coupled with scanning transmission electron microscopy (STEM) to map the Ag and Au elements of a AgNWs@ $\mathrm{TiO}_{2} @ \mathrm{GNS}$ nanowire with $30 \mathrm{~nm}$ GNS (Figure S2). The respective mappings and profiles through the nanowire are 
consistent with the expected nanostructure. Furthermore, the thickness of GNS can be controlled via a continuous addition of gold precursor.

The thinnest GNS sample with $30 \mathrm{~nm}$ exhibits no apparent plasmon response (Figure S3a), which is attributed to immature formation stage of Ag-Au hybrid nanostructures. ${ }^{26}$ Typical samples with 45 and $60 \mathrm{~nm}$ thick GNS are presented in Figure 2a-2b. Their extinction spectra are blue shifted from 574 to $559 \mathrm{~nm}$ as the GNS thickness increases (Figure 2c), which is consistent to previous reports on gold nanotube. ${ }^{26-27}$ Simulation of the electric field distributions are shown in Figure S3b-3d which display strong field enhancement at interface and surface of shell. In addition, the theoretical extinction spectra were calculated via FDTD to quantify the effects of GNS on the optical response of the hybrid nanowires (Figure 2d). During the simulation, Johnson and Christy's data were used and the silver dielectric function was fixed with a uniform surrounding medium $(n=1.33)$. From the simulations presented in Figure 2d, it can clearly be seen that the position of the extinction peaks is basically in qualitative agreement with the experimental UV/Vis spectra. The dipole-dipole coupling effect results in a slightly red-shifted and broader peaks in the experimental observation. These samples with different GNS thicknesses were used to study their ultrafast dynamics performance due to their excellent plasmonic behavior.

\section{Ultrafast Dynamics of Hybrids Nanostructure with Interband Excitation}

Visible pump-probe transient absorption measurements were utilized to investigate the carrier relaxation dynamics of plasmonic hybrid structures. Figure 3 shows their optical density change $(\Delta \mathrm{OD})$ spectrum and temporal evolution. $\Delta \mathrm{OD}$ is the difference of the material's absorption in wavelength range with and without the pump pulse excitation. All the hybrid nanostructures were excited in the interband transition region with $3.88 \mathrm{eV}$ photo energy and $3 \mathrm{~mW}$ pump power. Figure 3a shows the two-dimensional contour maps (combined with the corresponding spectral and 
temporal dynamics) of the sample with the 45-nm GNS. In this frequency domain the spectrum does not show any conventional photobleaching signal around the plasmonic peak (located at $574 \mathrm{~nm}$ as in the steady-state measurement). Corresponding spectra with various delay times are shown in Figure 3b. In the considered time domain in Figure 3c, the decay can be well-fitted by using two components: a fast femtosecond decay and slow picosecond one. The slow 2.86 picoseconds decay lifetime component which represents the relaxation dynamics of the electron-phonon coupling is consistent with previous reports regarding the electron-phonon coupling for gold nanospheres and nanorods. ${ }^{13}$ However, the relaxation also exhibits a much faster decay governed by 74.09 fs with a large pulsewidth-limited ultrafast component (pump laser pulse is $100 \mathrm{fs}$ ). In the plasmonic ultrafast dynamics process, the electron-electron scattering happens in this timescale after an extremely short dephasing time below 10 fs. $^{24}$ The photoexcited electron-hole pairs first form non-thermal distribution, which cannot be described using Fermi-Dirac statistics. ${ }^{19}$ This distribution thermalizes and cools the electrons through electron-electron scattering collisions happen faster than $100 \mathrm{fs}$, subsequently electron-phonon coupling further cools electrons in the picoseconds timescale. Due to the extremely short timescale for electron-electron scattering, it is challenging to observe their dynamics in transient absorption measurement. ${ }^{6}$ Therefore, the Rayleigh scattering spectra are usually employed to estimate their electron surface scattering parameter under linewidth measurement of single plasmonic nanoparticles. ${ }^{28-29}$ Recently, a pulsewidth-limited ultrafast relaxation dynamics was reported in hybrid gold nanodisk, which is consist with our current observation. ${ }^{19-20}$ The efficient electron-electron scattering dominates the high energetic hot electrons relaxation whereas the electron-phonon coupling dominate the relaxation pathway for low-energy electrons near the Fermi sea. The theoretical simulation indicated that the thinner $(45 \mathrm{~nm})$ insulator was more efficient than the thicker $(60 \mathrm{~nm})$ one to generate more energetic hot electrons beyond 
Fermi energy. ${ }^{20}$ Our ultrathin $5 \mathrm{~nm}-\mathrm{TiO}_{2}$ layer provides a proper prerequisite for such an efficient energetic hot electron generation. Figure $3 \mathrm{~d}$ and Figure $3 \mathrm{e}$ show the color map and transient spectral measurement for the thicker GNS sample, which exhibits a similar behavior: no photobleaching signal and as well as a broad absorption. Figure $3 \mathrm{f}$ shows the temporal performance, which can also be described by two components: a fast decay with 47.89 fs and a slow one of 3.19 ps. Compared with the thinner sample (74 fs), the shorter electron-electron scattering time indicates slightly stronger collision effect, corresponding to a higher initial electron temperature after excitation. This leads to slower electron-phonon coupling rate (in the picosecond decay range) according to the two-temperature model. ${ }^{5}$ In general, the photoexcited electron-hole pairs can undergo different decay pathways including relaxation through electron-electron scattering or hot electron transfer. In the system discussed herein, the $\mathrm{TiO}_{2}$ dielectric layer could serve as an efficient acceptor, and the hot electron transfer process is desirable to occur. In addition, electron-electron scattering and hot electron transfer are two competing pathways during relaxation. The hybrids with the thinner GNS and weak electron-electron scattering effects could result in an efficient hot electron transfer process. On the other hand, the shorter electron-phonon coupling time also demonstrates the higher efficiency of the hot electron injection. ${ }^{30-31}$

\section{Ultrafast Dynamics of Hybrid Nanostructures with Intraband Excitation}

The ultrafast spectroscopy technique was also employed to examine the carrier relaxation for these hybrid plasmonic nanostructures under pumping energy in the intraband excitation. A similar measurement was conducted for both thin and thick GNS samples under $2.75 \mathrm{eV}$ pumping, with power of $3 \mathrm{~mW}$. Conventional photobleaching characteristics located around plasmonic mode at 560 $\mathrm{nm}$ for the thinner one (Figure 4a) and $546 \mathrm{~nm}$ for thicker one (Figure 4d) were observed, which was quite different from the interband excitation. It demonstrates that $2.75 \mathrm{eV}$ pump pulses have 
managed to excite a larger number of electron-hole pairs, which is similar to ground-state bleaching in semiconductors. ${ }^{13}$ The increased electron temperature would dramatically change both the real and imaginary part of dielectric function, ${ }^{32}$ leading to the slight shift of the bleaching peak (negative signal) and absorption peak wings (positive signal), as shown in Figure $4 \mathrm{~b}$ and Figure 4e. Finally, the relaxation dynamics of the excited carriers observed in temporal response shown in Figure $4 c$ and Figure $4 \mathrm{f}$ give electron-phonon coupling times of 0.81 and 1.24 ps for the thinner and thicker samples, respectively.

The pulsewidth-limited ultrafast relaxation with femtoseconds components is absent in both time-domain measurements. The shorter decay time $(0.81 \mathrm{ps})$ in the thinner GNS nanostructure sample suggests that it is possible to generate a more efficient hot-electron injection. This process would decrease the amount of energy absorbed by the nanostructures, lowering the initial electron temperature in plasmonic materials.

\section{Hot Electron Transition and Relaxation}

In frequency domain transient measurements, different optical transient responses are attributed to electron transition mechanisms with different pump wavelengths. It is notable that the $d$-band energy of $\mathrm{Ag}-\mathrm{Au}$ alloy was estimated around $3.0 \mathrm{eV}$ for the system based on dielectric function analysis (2.5 $\mathrm{eV}$ for $\mathrm{Au}$ and $4.0 \mathrm{eV}$ for $\mathrm{Ag}) .{ }^{33}$ The pump energies with 3.88 and $2.75 \mathrm{eV}$ correspond to alloy's interband and intraband transitions, as illustrated in Figure 5. Figure 5a shows an intraband transition between a filled state located $2.75 \mathrm{eV}$ below the Fermi energy $\left(E_{f}\right)$, and an empty state just above $E_{f}$. This process is consistent with a classical surface plasmon excitation, where the subsequent plasmon decays into electron-hole pairs and results in a massive hot electron generation just above the $E_{f}$. The plasmonic electrons relaxation back to the filled states below $E_{f}$ finally leads to the strong photobleaching decay behavior located in their plasmon energy. Our experimental observation shows 
a silent femtosecond electron-electron scattering component assuming then that the generated carriers are not energetic electrons but instead low energy carriers with energy close to the $E_{f}$. However, Figure $5 \mathrm{~b}$ shows that for an interband transition with extremely high pump energy of 3.88 $\mathrm{eV}$, the excess energy can produce more opportunities to generate the energetic hot electrons well above the $\mathrm{E}_{f}$. These high energy hot carriers are responsible for the pulsewidth-limited ultrafast femtosecond relaxation component, in contrast with the femtosecond decay absence under intraband excitation. Besides, $d$-band electrons under interband excitation do not perturb the intraband electrons transition of plasmon excitation.

Their plasmon resonance peak absorption exhibits no reduction after incident pump laser excitation, leading to silent photobleaching signal in transient absorption spectra. The broaden photo-induced absorption signals are also induced by the increase of the imaginary part of the dielectric function under high energy pumping. Figure 5c illustrates a possible hot electron transfer process with a metal contacting a $\mathrm{TiO}_{2}$ layer. Based on previous experimental analyses and simulation, the electron-electron scattering of excited carriers after optical pumping is a competing pathway against the hot electron injection to adjacent semiconductor conduction bands. ${ }^{30,34}$ The reason could be the photoexcited hot electrons would lose their energy suffering from a strong electron-electron scattering effect, leading to decrease the population of excited hot carriers with adequate energy to cross the Schottky energy barrier interface. In our case, it is obvious that the thin GNS sample shows the fastest electron-phonon coupling, which indicates an efficient hot electron transfer. In contrast, the thicker one shows a stronger electron-electron scattering process with a faster femtosecond component decay and this is not beneficial for the electron transfer into the $\mathrm{TiO}_{2}$ semiconductor pathway. Therefore, it is crucial to clarify the different electron transition processes in nanostructures in order to take advantage of the photo-induced hot electrons or holes for assisted 
specific photochemical applications. ${ }^{35-36}$

\section{CONCLUSION}

In summary, hybrid plasmonic nanostructures of AgNWs@ $\mathrm{TiO}_{2} @ \mathrm{GNS}$ with different GNS thicknesses have been synthesized from facile wet chemical approach. Their plasmonic behaviors were studied from steady-state absorption spectra. Optical properties of hot electrons in the plasmonic nanostructures were measured and their ultrafast dynamics characterized by transient absorption spectroscopy were discussed. The energetic electrons were shown to decay into two ultrafast electron-electron scattering channels on a femtosecond timescale. Our results also show that the selection of the pump light energy is crucial for the generation of efficient energetic hot electrons. The interband and intraband electron transition mechanisms determine the behaviors of the different hot electron dynamics. The insights involved here improved our understanding on efficient hot carrier generation and injection, which is essential for hot electron harvesting in ultrafast nanophotonics and photocatalysis applications.

\section{ASSOCIATED CONTENT}

The Supporting Information is available free of charge on the ACS Publications website

\section{AUTHOR INFORMATION}

\section{Corresponding Authors}

Email: tche@szu.edu.cn

Email: chen.r@sustc.edu.cn

Email: zktang@umac.mo 


\section{Notes}

The authors declare no competing financial interest.

\section{ACKNOWLEDGMENTS}

This work is supported by the research and development grant funding from University of Macau

(MYRG 2018-00142, FDCT 199/2017/A3), National Natural Science Foundation of China (11574130 and 11404161), and Shenzhen Science and Technology Innovation Committee (Projects Nos.: KQJSCX20170726145748464, JCYJ20150930160634263, and KQTD2015071710313656). R. C. acknowledges the support from National 1000 plan for Young Talents.

\section{REFERENCE}

1. Odom, T. W.; Schatz, G. C. Introduction to Plasmonics. Chem. Rev 2011, 111, 3667-3668.

2. Atwater, H. A.; Polman, A. Plasmonics for Improved Photovoltaic Devices. Nat. Mater 2010, 9 , 865.

3. Linic, S.; Christopher, P.; Ingram, D. B. Plasmonic-Metal Nanostructures for Efficient Conversion of Solar to Chemical Energy. Nat. Mater 2011, 10, 911.

4. Li, M.; Cushing, S. K.; Wu, N. Plasmon-Enhanced Optical Sensors: A Review. Analyst 2015, 140, 386-406.

5. Mayer, K. M.; Hafner, J. H. Localized Surface Plasmon Resonance Sensors. Chem. Rev 2011, $111,3828-3857$.

6. Hartland, G. V. Optical Studies of Dynamics in Noble Metal Nanostructures. Chem. Rev 2011, $111,3858-3887$.

7. Hartland, G. V.; Besteiro, L. V.; Johns, P.; Govorov, A. O. What's So Hot About Electrons in Metal Nanoparticles? ACS Energy Lett 2017, 2, 1641-1653.

8. Brongersma, M. L.; Halas, N. J.; Nordlander, P. Plasmon-Induced Hot Carrier Science and Technology. Nat. Nanotechnol 2015, 10, 25.

9. Linic, S.; Aslam, U.; Boerigter, C.; Morabito, M. Photochemical Transformations on Plasmonic Metal Nanoparticles. Nat. Mater 2015, 14, 567.

10. Knight, M. W.; Sobhani, H.; Nordlander, P.; Halas, N. J. Photodetection with Active Optical Antennas. Science 2011, 332, 702-704.

11. Aslam, U.; Chavez, S.; Linic, S. Controlling Energy Flow in Multimetallic Nanostructures for Plasmonic Catalysis. Nat. Nanotechnol 2017, 12, 1000.

12. Boerigter, C.; Aslam, U.; Linic, S. Mechanism of Charge Transfer from Plasmonic 
Nanostructures to Chemically Attached Materials. ACS Nano 2016, 10, 6108-6115.

13. Jain, P. K.; Qian, W.; El-Sayed, M. A. Ultrafast Electron Relaxation Dynamics in Coupled Metal Nanoparticles in Aggregates. J. Phys. Chem. B 2006, 110, 136-142.

14. Cushing, S. K.; Wu, N. Progress and Perspectives of Plasmon-Enhanced Solar Energy Conversion. J. Phys. Chem. Lett 2016, 7, 666-675.

15. Cushing, S. K.; Li, J.; Meng, F.; Senty, T. R.; Suri, S.; Zhi, M.; Li, M.; Bristow, A. D.; Wu, N. Photocatalytic Activity Enhanced by Plasmonic Resonant Energy Transfer from Metal to Semiconductor. J. Am. Chem. Soc 2012, 134, 15033-15041.

16. Li, J.; Cushing, S. K.; Meng, F.; Senty, T. R.; Bristow, A. D.; Wu, N. Plasmon-Induced Resonance Energy Transfer for Solar Energy Conversion. Nat. Photonics 2015, 9, 601.

17. Li, J.; Cushing, S. K.; Bright, J.; Meng, F.; Senty, T. R.; Zheng, P.; Bristow, A. D.; Wu, N. $\mathrm{Ag} @ \mathrm{Cu} 2 \mathrm{o}$ Core-Shell Nanoparticles as Visible-Light Plasmonic Photocatalysts. ACS Catal 2013, 3, 47-51.

18. Cushing, S. K.; Li, J.; Bright, J.; Yost, B. T.; Zheng, P.; Bristow, A. D.; Wu, N. Controlling Plasmon-Induced Resonance Energy Transfer and Hot Electron Injection Processes in Metal@Tio2 Core-Shell Nanoparticles. J. Phys. Chem. C 2015, 119, 16239-16244.

19. Sykes, M. E.; Stewart, J. W.; Akselrod, G. M.; Kong, X.-T.; Wang, Z.; Gosztola, D. J.; Martinson, A. B. F.; Rosenmann, D.; Mikkelsen, M. H.; Govorov, A. O., et al. Enhanced Generation and Anisotropic Coulomb Scattering of Hot Electrons in an Ultra-Broadband Plasmonic Nanopatch Metasurface. Nat. Commun 2017, 8, 986.

20. Harutyunyan, H.; Martinson, A. B. F.; Rosenmann, D.; Khorashad, L. K.; Besteiro, L. V.; Govorov, A. O.; Wiederrecht, G. P. Anomalous Ultrafast Dynamics of Hot Plasmonic Electrons in Nanostructures with Hot Spots. Nat. Nanotechnol 2015, 10, 770.

21. Rycenga, M.; Cobley, C. M.; Zeng, J.; Li, W.; Moran, C. H.; Zhang, Q.; Qin, D.; Xia, Y. Controlling the Synthesis and Assembly of Silver Nanostructures for Plasmonic Applications. Chem. Rev 2011, 111, 3669-3712.

22. Gargiulo, J.; Cerrota, S.; Cortés, E.; Violi, I. L.; Stefani, F. D. Connecting Metallic Nanoparticles by Optical Printing. Nano Lett 2016, 16, 1224-1229.

23. Zhang, S.; Geryak, R.; Geldmeier, J.; Kim, S.; Tsukruk, V. V. Synthesis, Assembly, and Applications of Hybrid Nanostructures for Biosensing. Chem. Rev 2017, 117, 12942-13038.

24. Besteiro, L. V.; Kong, X.-T.; Wang, Z.; Hartland, G.; Govorov, A. O. Understanding Hot-Electron Generation and Plasmon Relaxation in Metal Nanocrystals: Quantum and Classical Mechanisms. ACS Photonics 2017, 4, 2759-2781.

25. Govorov, A. O.; Zhang, H.; Gun'ko, Y. K. Theory of Photoinjection of Hot Plasmonic Carriers from Metal Nanostructures into Semiconductors and Surface Molecules. J. Phys. Chem. C 2013, 117, 16616-16631.

26. Sun, Y. Silver Nanowires - Unique Templates for Functional Nanostructures. Nanoscale 2010, 2 , 1626-1642.

27. Kohl, J.; Fireman, M.; O'Carroll, D. M. Surface Plasmon and Photonic Mode Propagation in Gold Nanotubes with Varying Wall Thickness. Phys. Rev. B 2011, 84, 235118.

28. Sönnichsen, C.; Franzl, T.; Wilk, T.; von Plessen, G.; Feldmann, J.; Wilson, O.; Mulvaney, P. Drastic Reduction of Plasmon Damping in Gold Nanorods. Phys. Rev. Lett 2002, 88, 077402.

29. Hu, M.; Novo, C.; Funston, A.; Wang, H.; Staleva, H.; Zou, S.; Mulvaney, P.; Xia, Y.; Hartland, G. V. Dark-Field Microscopy Studies of Single Metal Nanoparticles: Understanding the Factors That Influence the Linewidth of the Localized Surface Plasmon Resonance. J. Mater. Chem 2008, 18, 1949-1960. 
30. Ratchford, D. C.; Dunkelberger, A. D.; Vurgaftman, I.; Owrutsky, J. C.; Pehrsson, P. E. Quantification of Efficient Plasmonic Hot-Electron Injection in Gold Nanoparticle-Tio2 Films. Nano Lett 2017, 17, 6047-6055.

31. Wang, L.; Wang, Z.; Wang, H.-Y.; Grinblat, G.; Huang, Y.-L.; Wang, D.; Ye, X.-H.; Li, X.-B.; Bao, Q.; Wee, A.-S., et al. Slow Cooling and Efficient Extraction of C-Exciton Hot Carriers in Mos2 Monolayer. Nat. Commun 2017, 8, 13906.

32. Brown, A. M.; Sundararaman, R.; Narang, P.; Schwartzberg, A. M.; Goddard, W. A.; Atwater, H. A. Experimental and Ab Initio Ultrafast Carrier Dynamics in Plasmonic Nanoparticles. Phys. Rev. Lett 2017, 118, 087401.

33. Rioux, D.; Vallières, S.; Besner, S.; Muñoz, P.; Mazur, E.; Meunier, M. An Analytic Model for the Dielectric Function of Au, Ag, and Their Alloys. Adv. Opt. Mater 2014, 2, 176-182.

34. Wu, K.; Rodríguez-Córdoba, W. E.; Yang, Y.; Lian, T. Plasmon-Induced Hot Electron Transfer from the Au Tip to Cds Rod in Cds-Au Nanoheterostructures. Nano Lett 2013, 13, 5255-5263.

35. Zhao, J.; Nguyen, S. C.; Ye, R.; Ye, B.; Weller, H.; Somorjai, G. A.; Alivisatos, A. P.; Toste, F. D. A Comparison of Photocatalytic Activities of Gold Nanoparticles Following Plasmonic and Interband Excitation and a Strategy for Harnessing Interband Hot Carriers for Solution Phase Photocatalysis. ACS Cent. Sci 2017, 3, 482-488.

36. Schlather, A. E.; Manjavacas, A.; Lauchner, A.; Marangoni, V. S.; DeSantis, C. J.; Nordlander, P.; Halas, N. J. Hot Hole Photoelectrochemistry on Au@SiO $\mathrm{S}_{2} @ \mathrm{Au}$ Nanoparticles. J. Phys. Chem. Lett 2017, 8, 2060-2067. 


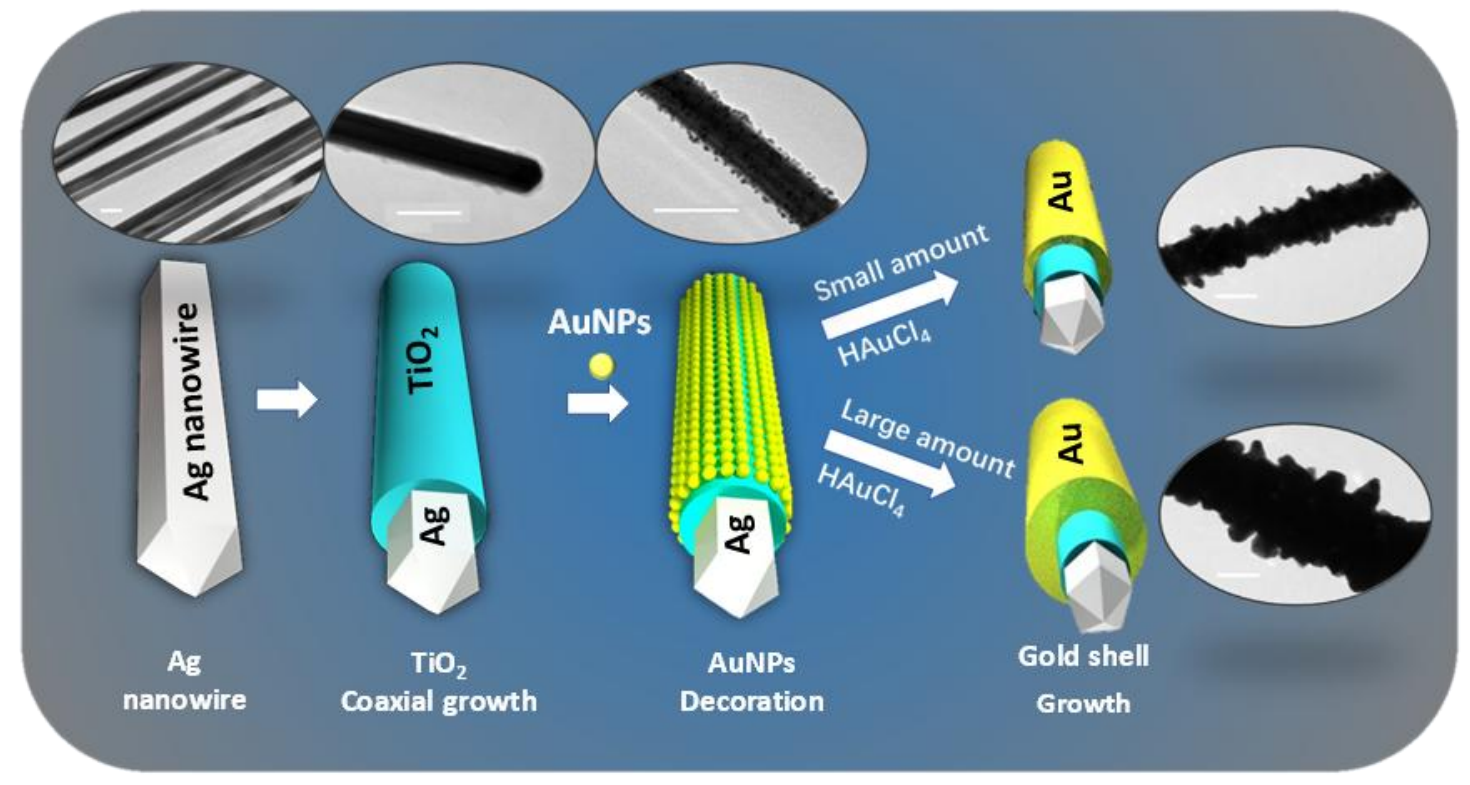

Figure 1. Schematic representative synthesis of the AgNWs@ $\mathrm{TiO}_{2} @ \mathrm{GNS}$ heterostructures with thin and thick GNS. White pentagonal prism stands for Ag, cyan coating stands for the $\mathrm{TiO}_{2}$ layer, and the light-yellow particles and walls are GNPs and GNS. The corresponding TEM images are around the schemes. All scale bars: $100 \mathrm{~nm}$. 

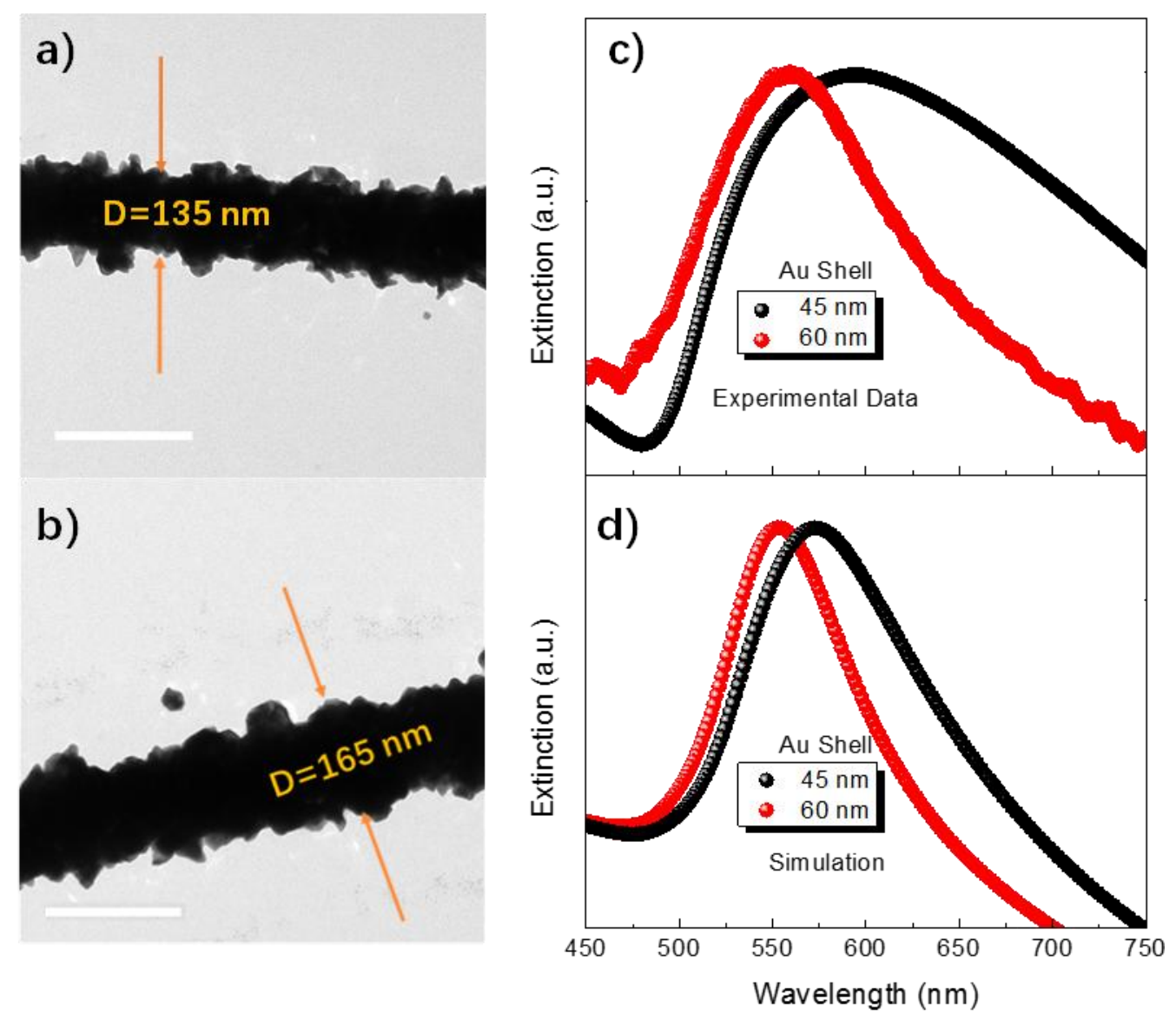

Figure 2. (a-b) Representatives TEM images of the nanoheterostructures and (c) experimental and (d) calculated extinction spectra of $\mathrm{AgNWs} / \mathrm{TiO}_{2} / \mathrm{GNS}$ with $45 \mathrm{~nm}$ and $60 \mathrm{~nm}$ thickness of GNS. All scale bars: $200 \mathrm{~nm}$. 

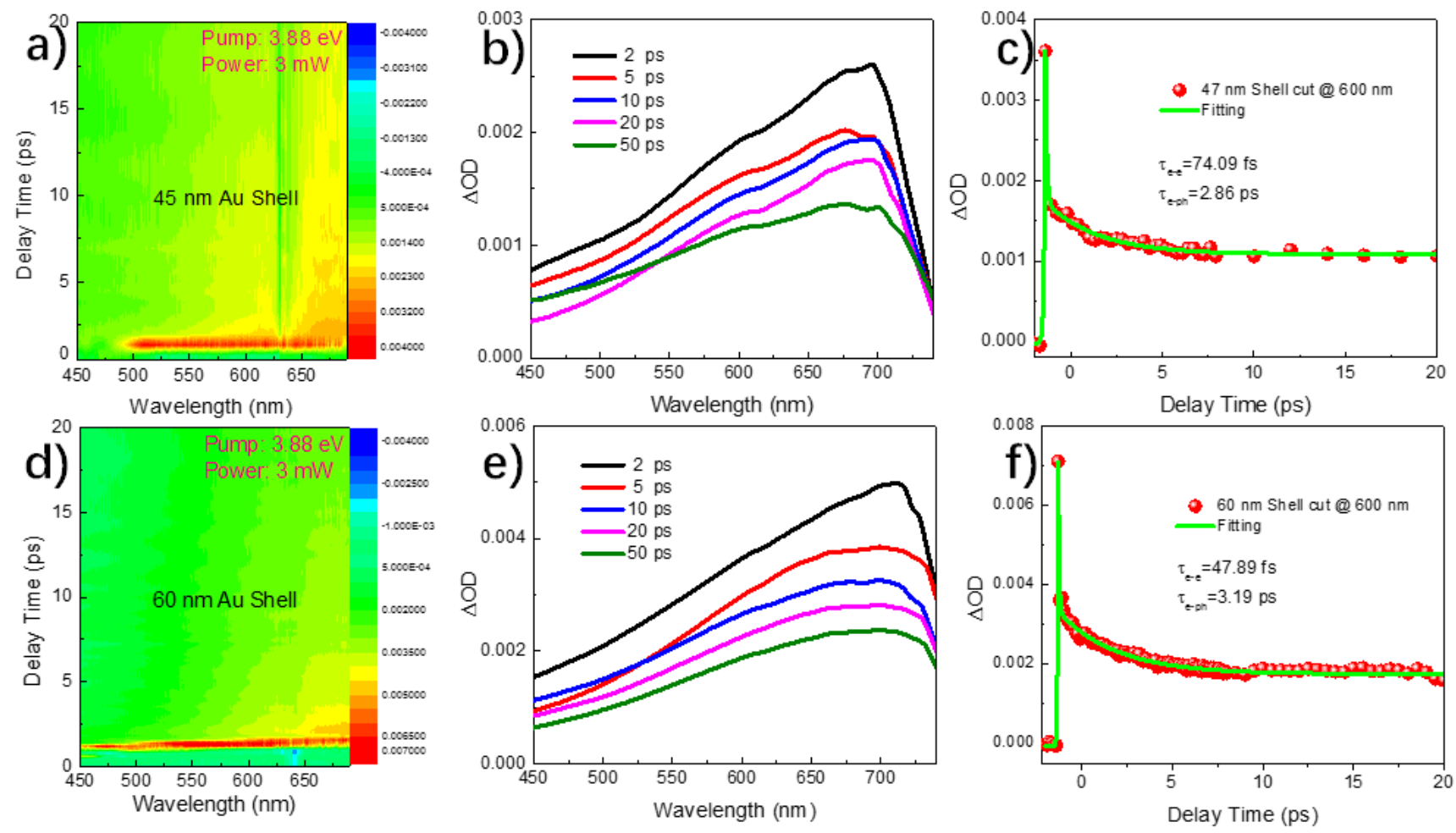

Figure 3. Transient absorption measurements of the $\mathrm{AgNW} @ \mathrm{TiO}_{2} @ \mathrm{GNS}$ with interband excitation. $\Delta$ OD spectral mapping of AgNWs@ $@ \mathrm{TiO}_{2}$ with $45 \mathrm{~nm}$ (a) and $60 \mathrm{~nm}$ (d) GNS. Transient absorption spectra at various time delays of AgNWs@ $\mathrm{TiO}_{2}$ with $45 \mathrm{~nm}$ (b) and $60 \mathrm{~nm}$ (e) GNS. Time dynamics of cut at $600 \mathrm{~nm}$ for AgNWs@ $\mathrm{TiO}_{2}$ with $45 \mathrm{~nm}$ (c) and $60 \mathrm{~nm}$ (f) GNS. All the experiments were performed under pump energy of $3.88 \mathrm{eV}$ with average power of $3 \mathrm{~mW}$. 

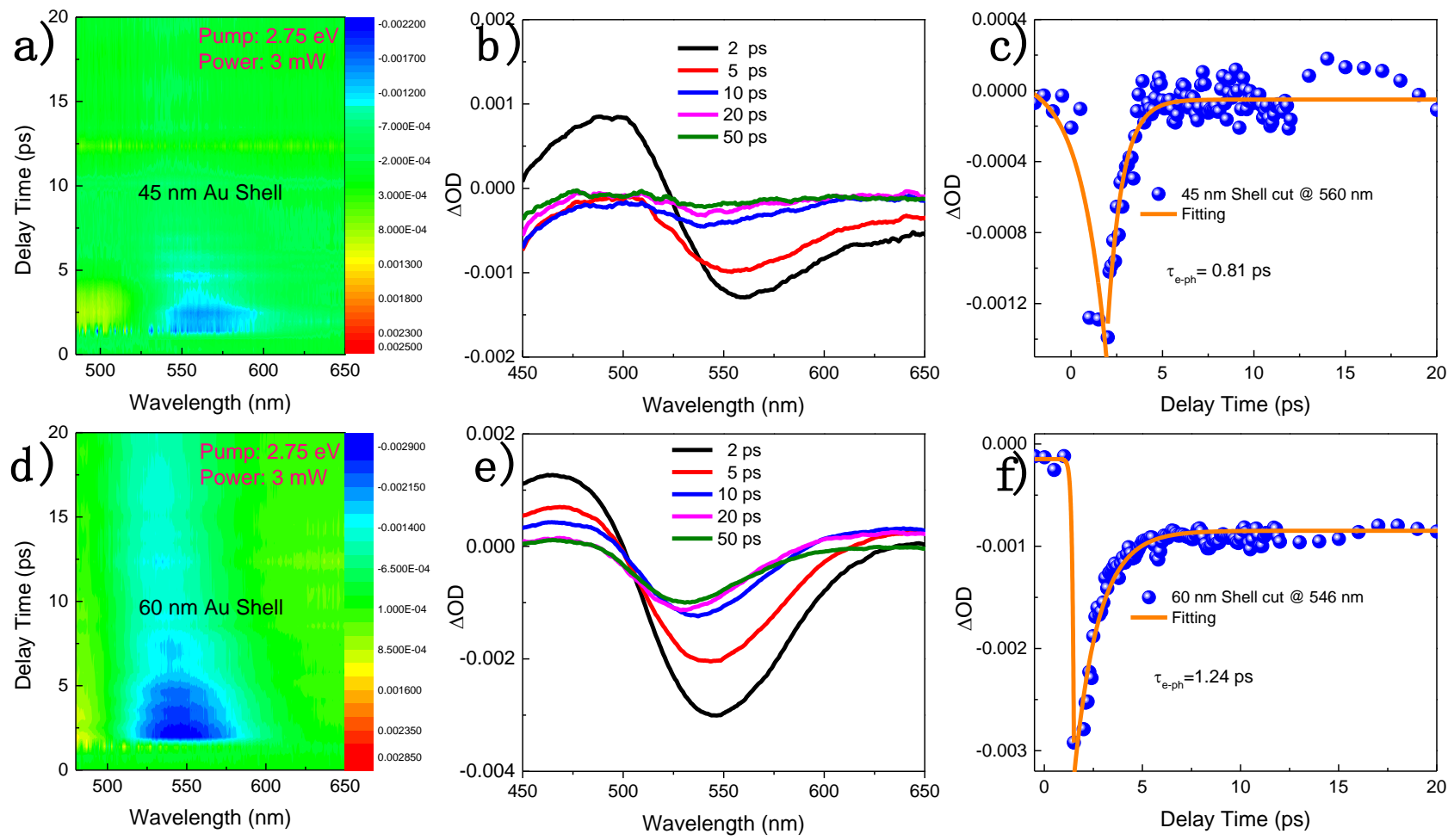

Figure 4. Transient absorption measurements of the $\mathrm{AgNW} @ \mathrm{TiO}_{2} @ \mathrm{GNS}$ with intraband excitation. $\Delta \mathrm{OD}$ spectral mapping of AgNWs@ $\mathrm{TiO}_{2}$ with 45 nm (a) and $60 \mathrm{~nm}$ (d) GNS. Transient absorption spectra at various time delays of AgNWs@ $\mathrm{TiO}_{2}$ with $45 \mathrm{~nm}$ (b) and $60 \mathrm{~nm}$ (e) GNS. Time dynamics of cut in photobleaching peaks for AgNWs@ $\mathrm{TiO}_{2}$ with $45 \mathrm{~nm}$ (c) and $60 \mathrm{~nm}$ (f) GNS. All the experiments were conducted under pump energy of $2.75 \mathrm{eV}$ with average power of $3 \mathrm{~mW}$. 
a)

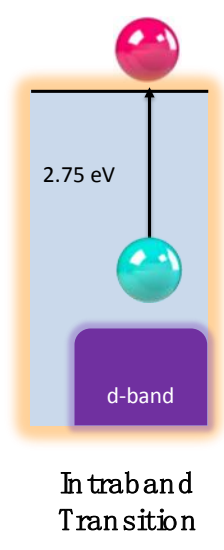

b)

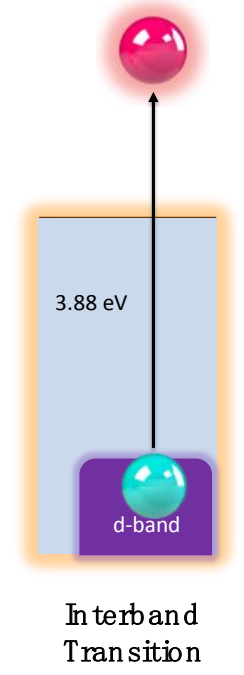

c)

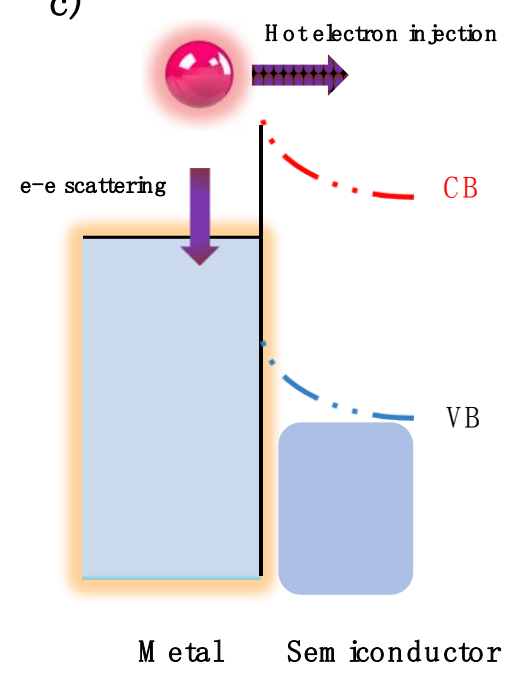

Figure 5. Different electron transition mechanisms described with energy diagrams. (a) Intraband excitation with $2.75 \mathrm{eV}$ to produce electrons near the Fermi surface. (b) Interband excitation with $3.88 \mathrm{eV}$ resulting in energetic electrons. (c) Hot electron transfer process against electron-electron scattering under formation of metal/semiconductor heterojunction. 


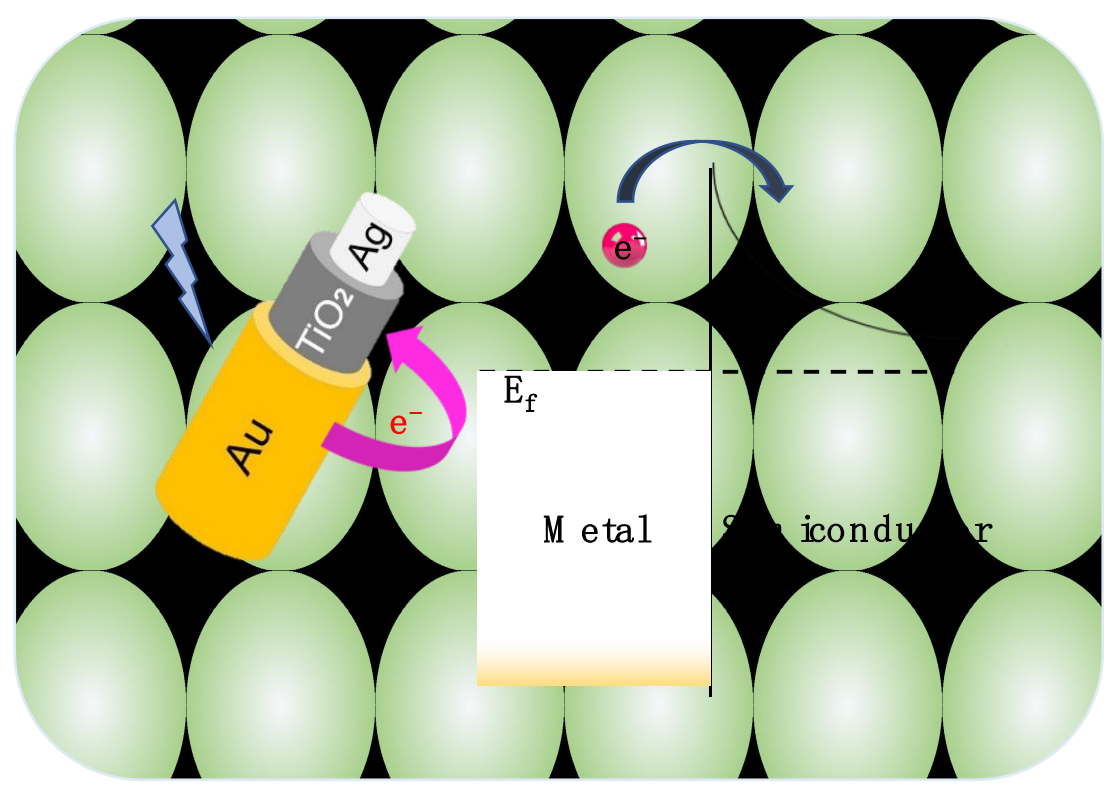

